\title{
AN UNSOLVABLE COUSIN PROBLEM
}

\author{
LEE A. RUBEL
}

(Communicated by Irwin Kra)

\begin{abstract}
We construct a Laurent series $\sum_{n=-\infty}^{\infty} A_{n} z^{n}$, convergent in an annulus $\{\rho<|z|<1 / \rho\}$ for some $\rho<1$, which satisfies an algebraic differential equation (ADE) there, but such that neither of the series $\sum_{n=0}^{\infty} A_{n} z^{n}$ and $\sum_{n=-\infty}^{-1} A_{n} z^{n}$ satisfies any ADE.
\end{abstract}

In [RUB], we constructed a Fourier series $u(\theta)=\sum_{n=-\infty}^{\infty} A_{n} e^{i n \theta}$ that satisfies an $\mathrm{ADE}$, but such that the truncated series $\sum_{n=0}^{\infty} A_{n} e^{i n \theta}$ satisfies no $\mathrm{ADE}$ on any interval. The construction used, as its main tool, the Riesz-Herglotz factorization of the function $e^{-10 z^{1 / 3}} \sin \pi z^{1 / 3} / \pi z^{1 / 3}$, where it was shown that the Blaschke product associated with this (obviously differentially algebraic) bounded analytic function in the right half-plane is not differentially algebraic (DA), where we say that a function is DA if it solves some nontrivial $\operatorname{ADE}, P\left(x, y, y^{\prime}, \ldots, y^{(n)}\right)=0$, where $P$ is a complex polynomial in its $n+2$ variables. Several applications of these results were obtained, notably a series $\sum a_{n} \cos n x$ that is DA, but such that $\sum a_{n} \sin n x$ (same $a_{n}$ ) is not DA on any interval. Also, it was shown that the solution of the Dirichlet problem for Laplace's equation in the unit disc need not be DA even though the boundary values are assumed to be DA. In that paper, the relevance of these results to analog computers was elaborated upon.

In this note, we obtain all the above results, with the exception of the one about there existing a DA bounded analytic function whose Blaschke product factor is not DA, by entirely different means, namely by consideration of the entire function

$$
\varphi(z)=\prod_{n=0}^{\infty}\left(1-\frac{\cosh z}{\cosh n}\right) .
$$

The properties of this function are in the literature (see [VAL and WIT]), but at the cost of only a little space, we can develop them here.

THEOREM. Given $\rho$ with $0<\rho<1$, there exists a Laurent series $L(z)=$ $\sum_{n=-\infty}^{\infty} A_{n} z^{n}$ which converges for $\rho<|z|<1 / \rho$ and satisfies there an algebraic differential equation, but neither of the series $\sum_{n=0}^{\infty} A_{n} z^{n}$ and $\sum_{n=-\infty}^{-1} A_{n} z^{n}$ satisfies any algebraic differential equation.

This may be regarded as saying that a certain additive Cousin problem cannot be solved by a general-purpose analog computer, since (see [LIR]), the outputs of such computers may be identified with the functions that are differentially algebraic, at

Received by the editors August 14, 1987.

1980 Mathematics Subject Classification (1985 Revision). Primary 35A25; Secondary 31A10.

This research was partially supported by a grant from the National Science Foundation. 
least in the analytic case. Here, the Cousin data is the function $L(z)$ in the annulus, and the problem is to represent it as the difference (equivalently, the sum) of two DA functions, the first being analytic inside the outer circle, and the second being analytic outside the inner circle.

PROOF. We will implicitly use throughout this paper the fact (see [OST] or [BOR]) that the sum, product, composition, compositional inverse, of DA functions is again DA. First of all, following a suggestion of Steven Bank, we show that $\varphi$ is DA. For $\varphi$ is an entire function of order 2 that vanishes on the rectangular lattice points $\{n+2 k \pi i: n, k \in \mathbf{Z}\}$, which is exactly the zero-set of Weierstrass's sigma-function $\sigma(z)$, another entire function of order 2 .

Hence $\varphi(z)$ differs from $\sigma(z)$ just by a factor $\exp P$, where $P$ is a polynomial. But (see [SAZ]), $\sigma(z)$ is DA, and consequently $\varphi(z)$ is DA. Let

$$
f(z)=\prod_{n=1}^{\infty}\left(1-\frac{z+1 / z}{2^{n}+2^{-n}}\right)
$$

By the above considerations, $f \in \mathrm{DA}$. Let

$$
g(z)=\prod_{n=1}^{\infty}\left(1-\frac{z}{2^{n}}\right), \quad h(z)=\prod_{n=1}^{\infty}\left(1-\frac{1}{z 2^{n}}\right) .
$$

Straightforward computation (see [VAL, p. 226]) shows that

$$
f(z)=C g(z) h(z), \quad \text { where } C=\prod_{n=1}^{\infty}\left(1+\frac{1}{2^{2 n}}\right)^{-1}
$$

(Just write

$$
\begin{aligned}
\left(1-\frac{z}{2^{n}}\right)\left(1-\frac{1}{z 2^{n}}\right) & =1+\frac{1}{2^{2 n}}-\frac{1}{2^{n}}\left(z+\frac{1}{z}\right) \\
& \left.=\left(1+\frac{1}{2^{2 n}}\right)\left[1-\frac{z+1 / z}{2^{n}+2^{-n}}\right] .\right)
\end{aligned}
$$

However, $g(z) \notin \mathrm{DA}$. There are numerous ways to see this (see [WIT] for example). Here is one way. Note that $g(z)$ satisfies the (Poincaré) functional equation $g(2 z)=(1-z) g(z)$. Writing $g(z)=\sum a_{n} z^{n}$, we have

$$
2^{n} a_{n}=a_{n}-a_{n-1}, \quad \text { or } \quad a_{n}=a_{n-1} /\left(1-2^{n}\right) \text {. }
$$

Note that the $a_{n}$ are rational numbers. Roughly, then, $a_{n} \sim 2^{-n(n-1) / 2}$, which by [POP, p. 79] are too small to be the coefficients of a DA function. To conclude the proof of the theorem, we just take logarithms after first removing a finite number of zeros from $g$ and $h$, that is let $\tilde{g}(z)=\prod_{n=N}^{\infty}\left(1-z / 2^{n}\right), \tilde{h}(z)=\prod_{n=N}^{\infty}\left(1-1 / z 2^{n}\right)$, and let $\tilde{f}(z)=\tilde{g}(z) \tilde{h}(z)$. Then $\tilde{f} \in \mathrm{DA}$ while $\tilde{g} \notin \mathrm{DA}$, and consequently $\tilde{h} \notin \mathrm{DA}$. Taking $N$ so large that $\tilde{g}$ and $\tilde{h}$ become zero-free in $\{\rho<|z|<1 / \rho\}$, we let

$$
\tilde{F}(z)=\log \tilde{f}(z), \quad \tilde{G}(z)=\log \tilde{g}(z), \quad \tilde{H}(z)=\log \tilde{h}(z) .
$$

Then $\tilde{F}$ is holomorphic and DA in $\{\rho<|z|<1 / \rho\}$, while $\tilde{G}$ is holomorphic in $\{|z|<1 / \rho\}$ and $\tilde{H}$ is holomorphic in $\{\rho<|z|\}$, and $\tilde{G} \notin \mathrm{DA}, \tilde{H} \notin \mathrm{DA}$. Writing

$$
\tilde{F}(z)=\sum_{n=-\infty}^{\infty} A_{n} z^{n}
$$


we have proved our result, since

$$
\tilde{F}(z)=\tilde{G}(z)+\tilde{H}(z) .
$$

ADDED IN PROOF. From the theory of elliptic functions, the theta-series

$$
S(x)=\sum_{n=-\infty}^{\infty} q^{n^{2}} x^{n}
$$

satisfies an algebraic differential equation in $x$ of the third order. However, as Pólya observed, (see [POL I, II]) the "half" theta-series

$$
\varphi(x)=\sum_{n=0}^{\infty} q^{n^{2}} x^{n},
$$

for $q$ rational and $0<q<1$ satisfies no algebraic differential equation in $x$. Thus, the main result of our paper follows directly from the classical literature.

\section{REFERENCES}

[BAN] Steven B. Bank, On the hypertranscendence of meromorphic solutions of certain functional equations, Proc. Royal Soc. Edinburgh 83A (1979), 45-54.

[BOR] Michael Boshernitzan and Lee A. Rubel, Coherent families of polynomials, Analysis 6 (1986), 339-389.

[LIR] Leonard L. Lipshitz and Le: A. Rubel, A differentially algebraic replacement theorem, and analog computability, Proc. Amer. Math. Soc. 99 (1987), 367-372.

[OST] Alexander Ostrowski, Über Dirichletsche Reihen und algebraische Differentialgleichungen, Math. Z. 8 (1920), 241-298.

[POL I] G. Pollya, Über das Anwachsen von ganzen Funktionen die einer Differentialgleichung genügen, Vierteljahrschrift Naturforsch. Gesellschaft Zürich 61 (1916), 531-545.

[POL II] __, Un pendant du théorème d'approximation de Liouville dans la théorie des équations différentielles, Enseign. Math. 19 (1917), 96-97.

[POP] Jan Popken, Über arithmetische Eigenschaften analytischer Funktionen, North-Holland, Amsterdam, 1935.

[RUB] Lee A. Rubel, Some mathematical limitations of the general-purpose analog computer, Adv. Appl. Math. 9 (1988), 22-34.

[SAZ] S. Saks and A. Zygmund, Analytic functions, Translated by E. J. Scott, Monografie Mat. 28, Polskie Towarzystwo Matematyczne, Warszawa-Wroclaw, 1952.

[VAL] G. Valiron, Fonctions analytiques, Presses Universitaires de France, Paris, 1954.

[WIT] H. Wittich, Bemerkung zu einer Funktionalgleichung von H. Poincaré, Arch. Math. 2 (1950), 90-95.

Department of Mathematics, 1409 W. Green Street, University of Illinois, URBANA, ILLINOIS 61801 